Vietnam Journal of Mechanics, VAST, Vol.31, No. 2 (2009), pp. 122-132

\title{
AN ENFORCED ESSENTIAL BOUNDARY CONDITION BY PENALTY METHOD IN THE ELEMENT-FREE GALERKIN (EFG) METHODS
}

\author{
Nguyen Hoai Son \\ University of Technical Education Ho Chi Minh City
}

\begin{abstract}
A meshless approach to the analysis of two-dimensional elasticity problems by the Element-Free Galerkin (EFG) method is presented. This method is based on moving least squares approximant (MLS). The unknown function of displacement $u(x)$ is approximated by moving least square approximants $u^{h}(x)$. These approximants are constructed by using a weight function, a monomial basis function and a set of nonconstant coefficients. A subdivision similar to finite element method is used to provide a background mesh for numerical integration. The essential boundary conditions are enforced by Penalty Method. The results are obtained for a two-dimensional problem using different EFG weight functions and compared with the results of finite element method and exact methods.
\end{abstract}

Keywords. weight function, penalty method, moving least squares, meshfree.

\section{INTRODUCTION}

As for now the finite element method has been a powerful tool for solving partial differential equations. It has successfully been applied for a large number of engineering applications, for example solid mechanics, structure mechanics, electro magnetism, geo mechanics, bio mechanics and so on. But for the last fifteen years a new mesh free method has been subject to extensive research.

The element free Galerkin (EFG) method is a meshless method for solving partial differential equations which uses only a set of nodal points and a CAD_like description of the body to formulate the discrete model. It has been used extensively for fracture problems and has yielded good results when adequate refinement is used near the crack tip.

In this paper, a meshless approach to the analysis of two-dimensional elasticity problems by the Element-Free Galerkin (EFG) method is presented. This method is based on moving least squares approximant (MLS) to construct the approximate function for the Galerkin weak-form. These approximations are constructed by using a weight function, a monomial basis function and a set of non-constant coefficients. A subdivision similar to finite element method is used to provide a background mesh for numerical integration. The essential boundary conditions are enforced by Penalty Method. In this study, EFG is applied to elastostatics analysis. Path test, plate with a central circular hole will be computed in this paper. The results are obtained for a two-dimensional problem using different 
EFG weight functions and compared with the results of exact methods. In addition, EFG method Matlab code also is offered in this paper. This Matlab code can be developed to meshfree application software or other meshfree method in the further.

\section{MLS APPROXIMATIONS FUNCTIONS}

MLS functions were developed by Lancaster and Salkauskas to approximate curves and surfaces. We approximate the displacement field by a discrete sum

$$
u(x) \cong u^{h}(x)=\mathbf{p}^{T}(x) \mathbf{a}(x) \quad \forall x \in \Omega_{\mathbf{x}}
$$

where $p(x)$ is a linearly independent basis of $m$ functions,

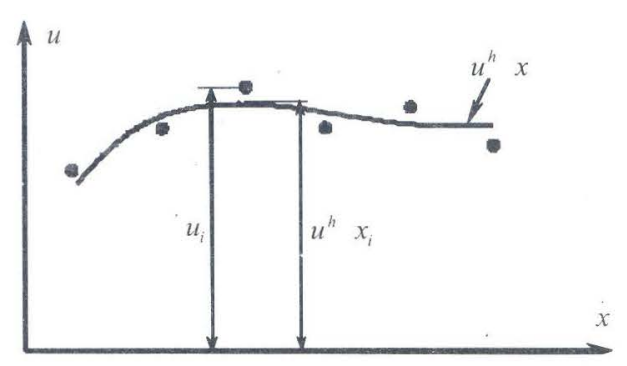

Fig. 1. The approximation function $u^{k}(x)$ and the nodal parameters $u_{i}$ in the MLS approximation

$$
p^{T}(x)=\left[\begin{array}{llll}
p_{0}(x) & p_{1}(x) & \ldots & p_{m}(x)
\end{array}\right]
$$

and $\mathbf{a}(x)$ is collection of undetermined parameters of the approximation

$$
a^{T}(x)=\left[\begin{array}{llll}
a_{0}(x) & a_{1}(x) & \ldots & a_{m}(x)
\end{array}\right],
$$

where each term is a function of the position $x \in \Omega$ (see Fig. 1).

The parameters $\mathbf{a}(x)$ are found at any $x$ point by minimizing the following weighted least squares discrete $L_{2}$ error norm (Nayroles-1992),

$$
J=\sum_{I=1}^{n} \omega\left(x-x_{I}\right)\left[u^{h}\left(x_{I}, x\right)-u_{I}\right]^{2}=\sum_{I=1}^{n} \omega\left(x-x_{I}\right)\left[p^{T}\left(x_{I}\right) a(x)-u_{I}\right]^{2}
$$

where $\omega\left(x-x_{I}\right)$ is a weighting function which is nonzero on the influence domain of the node $x_{I}$, thus generating a local approximation and sparse matrices. Only the $x_{I}$ nodes whose influence domains contain the $x$ point will appear in the sum (4). The dimension of the influence domain of each node and the choice of the weighting function are decisive parameters for the approximation by MLS [4,1].

Minimizing $J$ in order to the unknown parameters a $(x)$ results in

$$
A(x) a(x)=B(x) u \quad \text { or } \quad a(x)=A^{-1}(x) B(x) u
$$

with:

$$
A(x)=\sum_{I=1}^{n} \omega\left(x-x_{I}\right) p\left(x_{I}\right) p^{T}\left(x_{I}\right)
$$




$$
B(x)=\left[\begin{array}{llll}
\omega\left(x-x_{1}\right) p\left(x_{1}\right) & \omega\left(x-x_{2}\right) p\left(x_{2}\right) & \ldots & \omega\left(x-x_{n}\right) p\left(x_{n}\right)
\end{array}\right]
$$

Substituting the result (5) for $\mathbf{a}(x)$ in the initial approximation (1), this expression can be written in the usual form

$$
u^{h}(x)=\sum_{I=1}^{n} \Phi_{I}(x) u_{I}=\Phi(x) U
$$

where the shape function is defined by

$$
\Phi_{I}(x)=\sum_{j=0}^{m} p_{j}(x)\left(A^{-1}(x) B(x)\right)_{j I}=p^{T} A^{-1} B_{I},
$$

here $m$ is the order of the polynomial $p(x)$. To determine the derivatives from the displacement (8), it is necessary to obtain the shape function derivatives. The partial derivatives of the shape functions are obtained by

$$
\Phi_{I, x}=\left(p^{T} A^{-1} B_{I}\right)_{, x}=p_{, x}^{T} A^{-1} B_{I}+p_{, x}^{T}\left(A^{-1}\right)_{, x} B_{I}+p_{T} A^{-1} B_{I, x}
$$

where

$$
\begin{aligned}
& B_{I, x}(x)=\frac{d \omega}{d x}\left(x-x_{I}\right) p\left(x_{I}\right) \\
& A_{, x}^{-1}(x)=-A^{-1} A_{, x} A^{-1} \\
& A_{, x}=\sum_{I=1}^{n} \omega_{, x}\left(x-x_{I}\right) p\left(x_{I}\right) p^{T}\left(x_{I}\right) .
\end{aligned}
$$

It should be noted that EFG shape functions do not satisfy the Kronecker delta criterion: $\Phi_{I}(x) \neq \delta_{i j}$. Therefore they are not interpolants and the name approximation is used. So $u^{h}\left(x_{I}\right) \neq u_{I}$, the nodal parameters $u_{I}$ are not the nodal values of $u^{h}\left(x_{I}\right)$. The approximation to the displacement at the $I^{\text {th }}$ node depends on the nodal parameter $u_{I}$ as well as the nodal parameters $u_{I}$ through $u_{n}$ corresponding to all other nodes within the domain of influence of node $I$. This property makes the imposition of essential boundary conditions more complicated than with finite elements [6]. We will use Penalty method to enforce the essential boundary conditions.

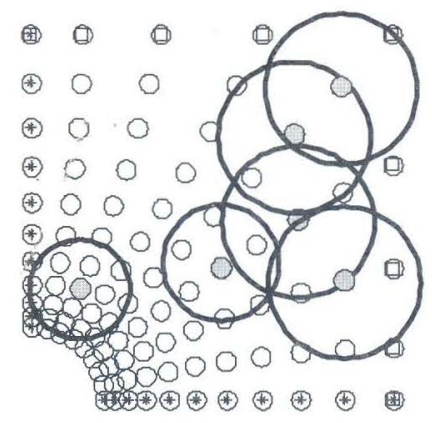

Fig. 2. Shape of the support domain

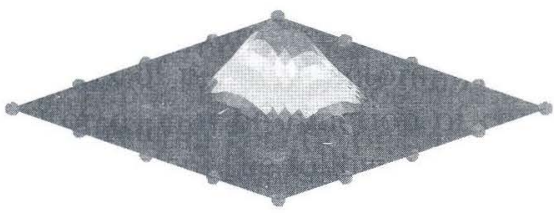

Fig. 3. Shape of the weight function 


\section{CHOICE OF SUPPORT DOMAIN AND WEIGHT FUNCTION}

There is no difference if circular or rectangular support domains are used in the EFG method $[1,2,4]$. The following implementations is made with circular. The weight function plays an important role for the EFG method (see Figs. 2, 3). A proper constructed weight function will give unique solutions when we determine the coefficient vector a. Weight functions need to have the following properties:

- Compact support, i.e. zero outside the support domain.

- Adopt positive values for all points in the support domain

- Have its maximum value at the current point and decrease when moving outwards.

There are many kinds of functions satisfying these properties, but the one used in this paper is a quartic spline function $[3,4,6]$

$$
\omega_{j}(r)=\left\{\begin{array}{ccl}
\frac{2}{3}-4 r_{j}^{2}+4 r_{j}^{3} & \text { when } \quad r_{j} \leq \frac{1}{2} \\
\frac{4}{3}-4 r_{j}+4 r_{j}^{2}-\frac{4}{3} r_{j}^{3} & \text { when } & \frac{1}{2} \leq r_{j} \leq 1 \\
0 & \text { when } & r_{j} \geq 1
\end{array}\right.
$$

with:

$$
r_{j}=\frac{\left\|x-x_{j}\right\|}{d_{\max } c_{j}},
$$

where $d_{\max }$ is a scaling parameter which is typically $2 \div 4$ [4] for a static analysis. The distance $c_{j}$ is determined by searching enough neighbor nodes for $A$ to be regular. Referring to (6), it can easily be seen that the matrix $A$ will be singular if a given node has only one neighbor in its domain of influence. In one dimension with a linear basis, the distance $c_{j}$ at a node is the maximum distance to the nearest neighbor, insuring that each node will have at least two neighbors in its domain of influence.

\section{WEAK FORM FOR SOLID MECHANICS}

The partial differential equation that controls solid mechanics for 2-D can be stated as:

$$
R^{T} \sigma+b=0
$$

This equation applies for all points in the problem domain $\Omega$. For the essential boundary, $S_{u}$, we have a prescribed displacement

$$
u=\bar{u} \text {. }
$$

For the natural boundary, $S_{t}$, we have a prescribed force given by the traction vector

$$
\bar{t}=S_{\sigma} n,
$$


where $n$ is the normal vector to the boundary and $S_{\sigma}$ is the stress tensor. The matrices in the formulation are given by

$$
\begin{aligned}
& R=\left[\begin{array}{cc}
\frac{\partial}{\partial x} & 0 \\
0 & \frac{\partial}{\partial y} \\
\frac{\partial}{\partial y} & \frac{\partial}{\partial x}
\end{array}\right] ; \quad \sigma=\left[\begin{array}{l}
\sigma_{x x} \\
\sigma_{y y} \\
\sigma_{x y}
\end{array}\right] ; \quad b=\left[\begin{array}{c}
b_{x} \\
b_{y}
\end{array}\right] ; \\
& u=\left[\begin{array}{l}
u_{x} \\
u_{y}
\end{array}\right] ; \quad n=\left[\begin{array}{l}
n_{x} \\
n_{y}
\end{array}\right] ; \quad S_{\sigma}=\left[\begin{array}{cc}
\sigma_{x x} & \sigma_{x y} \\
\sigma_{y x} & \sigma_{y y}
\end{array}\right]
\end{aligned}
$$

$R$ : operator working on the stress; $\sigma$ : stress matrix; $b$ : forces working on the body; $u$ : displacement vector for a point; $n$ : normal vector to a boundary point; $S_{\sigma}$ : stress tensor.

This is the strong form of the problem. In general we can not solve this equation analytically. This is why we need numerical methods like the finite element method. In order to solve the problem we need the regularity of the function $u$. There are several methods for doing this, the one used in this report is a variational principle. We construct the lagrangian function $L$ in solid mechanics

$$
L=T-\Pi+W,
$$

where $T$ is the kinetic energy, $\Pi$ is the elastic energy and $W$ is the work done by the external forces. The components of the function $L$ are calculated as

$$
\begin{gathered}
T=\frac{1}{2} \int_{\Omega} \rho \dot{u}^{T} \dot{u} d \Omega \\
\Pi=\frac{1}{2} \int_{\Omega} \varepsilon^{T} \sigma d \Omega \\
W=\int_{\Omega} u^{T} b d \Omega+\int_{S_{t}} u^{T} t d S_{t}
\end{gathered}
$$

\section{PENALTY METHOD}

The penalty method is another alternative to impose essential boundary conditions, which was first proposed by [2]. A detailed illustration is given by Zhu for the case of 2D linear elastostatics. Consider the same problem. This will lead to a modified Lagrange function $[3]$

$$
\bar{L}=L+\frac{\alpha}{2} \int_{S_{u}}(u-\bar{u})^{T}(u-\bar{u}) d S_{u},
$$

where $\alpha=\left[\begin{array}{llll}\alpha_{1} & \alpha_{2} & \ldots & \alpha_{k}\end{array}\right]$ is a diagonal matrix of penalty factors, with $k=2$ for $2 \mathrm{D}$ cases and $k=3$ for $3 \mathrm{D}$ cases. The penalty factor $\alpha_{i}$ can be function of coordinates and they can be different from each other. This report prefers the following simple method for determining the penalty facter:

$$
\alpha=10^{2}-10^{13} \times \max \text { (diagonal elements in the stiffness matrix). }
$$

It has also been suggested to use $\alpha=10^{2}-10^{8} \times$ Young's modulus matrix 
Taking $\delta \bar{L}=0$, we have the following algebraic equations

$$
\left(K-\alpha K^{u}\right) U=f-f^{u},
$$

where

$$
\begin{aligned}
& K_{I J}=\int_{\Omega} B_{I}^{T} D B_{J} d \Omega ; \quad f_{I}=\int_{\Omega} \Phi^{T} b d \Omega+\int_{S_{t}} \Phi^{T} t d S_{t} ; \\
& K_{I J}^{u}=\int_{S_{u}} N_{I} S N_{J} d S_{u} ; \quad f_{I}^{u}=\int_{S_{u}} N_{I} S \bar{u} d S_{u} . \\
& S=\left[\begin{array}{ll}
S_{x} & 0 \\
0 & S_{y}
\end{array}\right] ; \quad S_{i}= \begin{cases}1 & \text { if } u_{i} \in S_{u} \\
0 & \text { if } u_{i} \not \subset S_{u}, \quad i=1,2\end{cases}
\end{aligned}
$$

\begin{tabular}{|c|}
\hline PRE PROCESSING \\
\hline $\begin{array}{l}\text { - Generate nodes } \\
\text { - Generate integration points and cells } \\
\text { - Set variables and constants }\end{array}$ \\
\hline MAIN PROGRAM \\
\hline $\begin{aligned} \text { for } i=1 \text { : all integration points } \\
\text { for } j=1 \text { all nodes } \\
\text { if } j \in \text { support domain for } i \\
\text { end if } \\
\text { end for } \\
=\text { Calculate shape function } \\
\text { - Assemble to global system matrices }\end{aligned}$ \\
\hline $\begin{array}{l}\text { end for } \\
\qquad \text { Solve equations system }\end{array}$ \\
\hline POST PROCESSING \\
\hline $\begin{array}{l}\text { for }=1 \text {. number of output points } \\
\quad \text { Determine nodes inside support domain to i. } \\
\quad \text { Calculate real displacement } \\
\text { - Compute strains and stresses for } i \text {. } \\
\text { end for }\end{array}$ \\
\hline
\end{tabular}

General algorithm for EFG with Gauss integration is following.

\section{NUMERICAL EXAMPLE}

The global error indicator, the $L_{2}$-norm error in displacement, is defined by [5]

$$
L_{2}=\frac{\left\{\sum_{j=1}^{N}\left\{\left(u_{j}^{\text {num }}-u_{j}^{\text {exact }}\right)^{2}+\left(v_{j}^{\text {num }}-v_{j}^{\text {exact }}\right)^{2}\right\}\right\}^{1 / 2}}{\left\{\sum_{j=1}^{N}\left\{\left(u_{j}^{\text {exact }}\right)^{2}+\left(v_{j}^{\text {exact }}\right)^{2}\right\}\right\}^{1 / 2}}
$$


The $L_{2}$-norm error is a better measure of the overall performance of a numerical model than the conventional relative error for a single point. In this work, we will use the global $L_{2}$-norm error as a measure of the overall performance of the numerical method.

Consider a plate with a central circular hole subjected to a unidirectional tensile load in the $x$ direction as shown in Fig. 4. Due to symmetry, only the upper right quadrant of the plate is modelled (see, Fig. 5). Symmetry conditions are imposed on the left and bottom edges. The inner boundary is traction free. Plane strain conditions are assumed

- The material constants: $E=2.10^{11} \mathrm{~N} / \mathrm{m}^{2}$

- The unidirectional tensile load: $q=1 \mathrm{~N} / \mathrm{m}$

- The demension of the plate: $L \times L=10 \times 10 \mathrm{~m} ; d=2 \mathrm{~m}$

$-\nu=0.3$

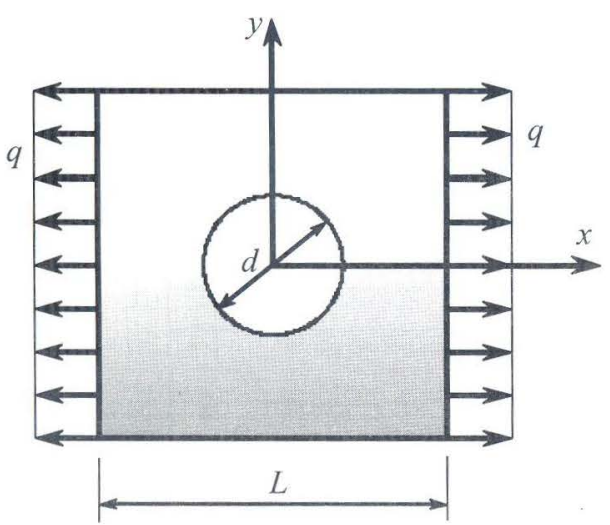

Fig. 4. A 2D solid with a central hole subjected to a unidirectional tensile load

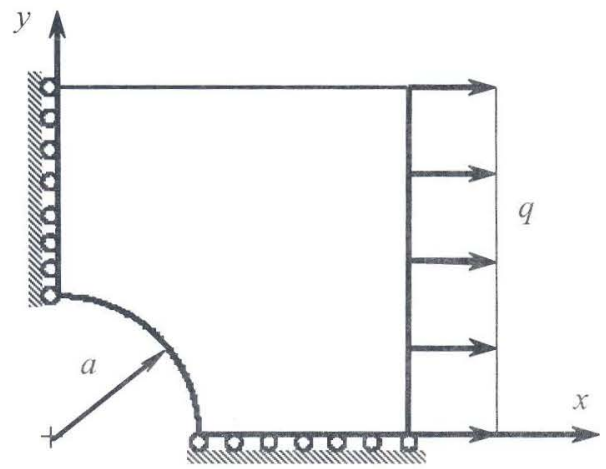

Fig. 5. One quarter model of plate

The analytical solution for the stresses of an infinite plate [6] is of the form

$$
\begin{gathered}
\sigma_{x}=\left[1-\frac{a^{2}}{r^{2}}\left(\frac{3}{2} \cos 2 \theta+\cos 4 \theta\right)+\frac{3}{2} \frac{a^{4}}{r^{4}} \cos 4 \theta\right] q \\
\sigma_{y}=\left[-\frac{a^{2}}{r^{2}}\left(\frac{1}{2} \cos 2 \theta-\cos 4 \theta\right)-\frac{3}{2} \frac{a^{4}}{r^{4}} \cos 4 \theta\right] q \\
\sigma_{x y}=\left[-\frac{a^{2}}{r^{2}}\left(\frac{1}{2} \sin 2 \theta+\sin 4 \theta\right)+\frac{3}{2} \frac{a^{4}}{r^{4}} \sin 4 \theta\right] q \\
u_{r}=\frac{1}{4 G}\left\{r\left(\frac{k-1}{2}+\cos 2 \theta\right)+\frac{a^{2}}{r}[1+(1+k) \cos 2 \theta]-\frac{a^{4}}{r^{3}} \cos 2 \theta\right\} \\
u_{\theta}=\frac{1}{4 G}\left[(1-k) \frac{a^{2}}{r}-r-\frac{a^{4}}{r^{3}}\right] \sin 2 \theta
\end{gathered}
$$

with:

$$
G=\frac{E}{2(1+\nu)}, \quad k=3-4 \nu \quad \text { for } \quad \sigma_{z}=0
$$

where $(r, \theta)$ are the polar coordinates and $\theta$ is measured counterclockwise from the positive $x$ axis. The analytical results are employed as the reference results for comparison [6]. 
The arrangement of nodes and quadrature cells is shown in Figure 6. In each quadrature cell, $4 \times 4$ Gauss points are used. The solutions are obtained using a quadratic basic function with cubic spline weight function.

Table 1. Comparison stress $\sigma_{x}$ at $(0, r)$

\begin{tabular}{|c|c|c|c|}
\hline $\begin{array}{c}\text { Influence } \\
\text { radius } r_{i}\end{array}$ & $\begin{array}{c}\text { Stresses } \sigma_{x} \text { at }(0, r) \\
\text { of exact solution }\end{array}$ & $\begin{array}{c}\text { Stresses } \sigma_{x} \text { at }(0, r) \text { of } \\
\text { EFG solution }\end{array}$ & $\begin{array}{c}\text { Error } \\
(\%)\end{array}$ \\
\hline 1.0 & 3.0 & 2.7342 & 8.8590 \\
\hline 1.1 & 3.0 & 3.0843 & 2.8094 \\
\hline 1.2 & 3.0 & 3.0572 & 1.9062 \\
\hline 1.3 & 3.0 & 3.0465 & 1.5507 \\
\hline 1.4 & 3.0 & 3.0388 & 1.2932 \\
\hline 1.5 & 3.0 & 3.0268 & 0.8950 \\
\hline 1.6 & 3.0 & 3.0225 & 0.7505 \\
\hline 1.7 & 3.0 & 3.0438 & 1.4604 \\
\hline 1.8 & 3.0 & 3.0826 & 2.7543 \\
\hline 1.9 & 3.0 & 3.1114 & 3.7135 \\
\hline 2.0 & 3.0 & 3.1205 & 4.0154 \\
\hline 2.1 & 3.0 & 3.1243 & 4.1421 \\
\hline
\end{tabular}

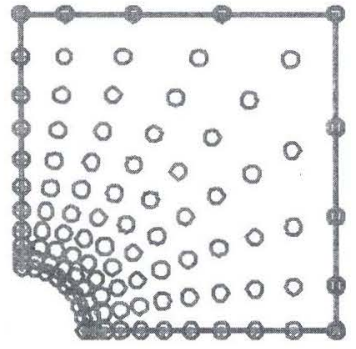

Fig. 6. Nodal arrangement

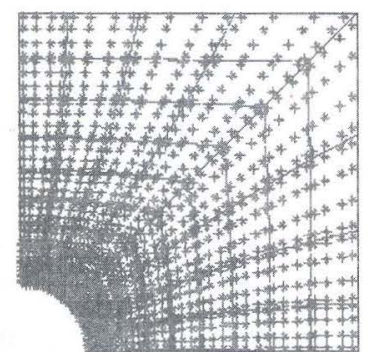

Fig. 8. Quadrature cells and Gauss points

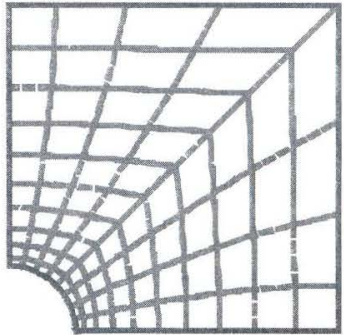

Fig. 7. Mesh used for integration

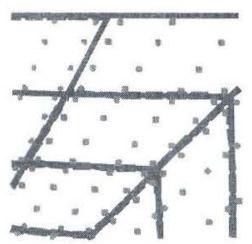

Fig. 10 presents the distribution of normal stress $\sigma_{x}$ along $x=0$ of the $7 \times 10$-node model with dimension of the influence domain $r_{i}=1.2$ and $r_{i}=1.6$. 


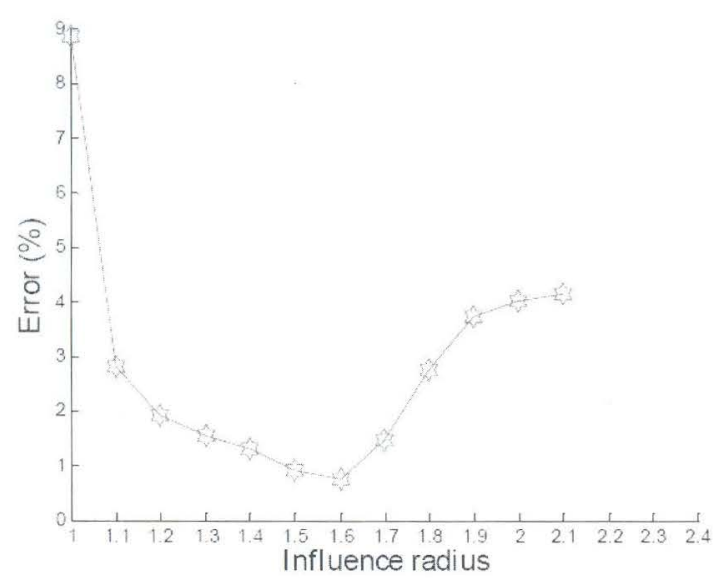

Fig. 9. Comparison between the exact and EFG solution for $\sigma_{x}$ with influence radius $r_{i}=1 \div 2.1$

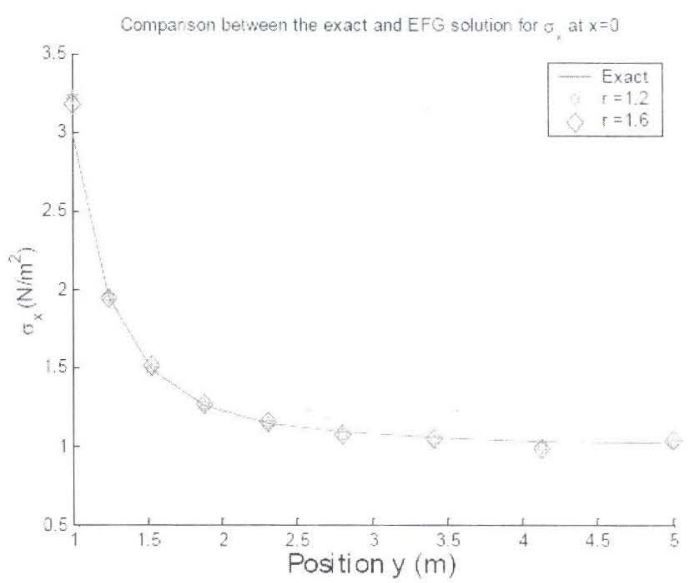

Fig. 10. Comparison between the exact and EFG solution for $\sigma_{x}$ at $x=0$ with influence radius $r_{i}=(1.2,1.6)$

Figure 11 presents the distribution of normal stress $\sigma_{x}$ along $x=0$ of the $7 \times 10$-node model with number of nodes $7 \times 10$ and $12 \times 14$. basis.

Fig. 12 shows the deformed meshless model, using spline weight function and linear

Fig. 13 and 14 show the contour plots of $\sigma_{x}$ stress distribution from the EFG method

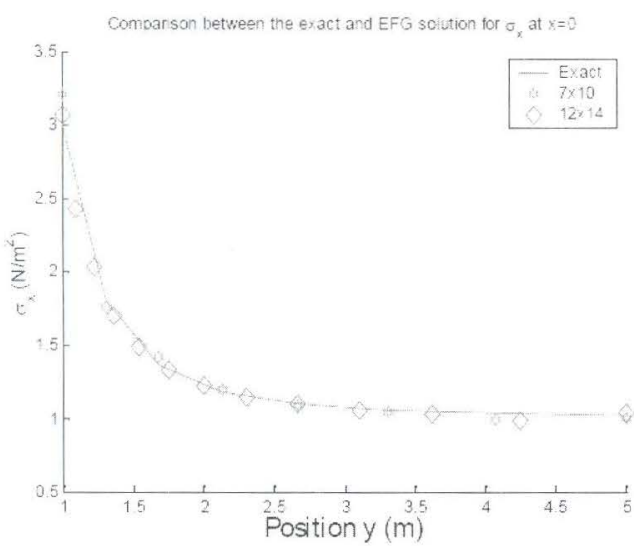

Fig. 11. Comparison between the exact and EFG solution for at with number of nodes $(7 \times 10,12 \times 14)$

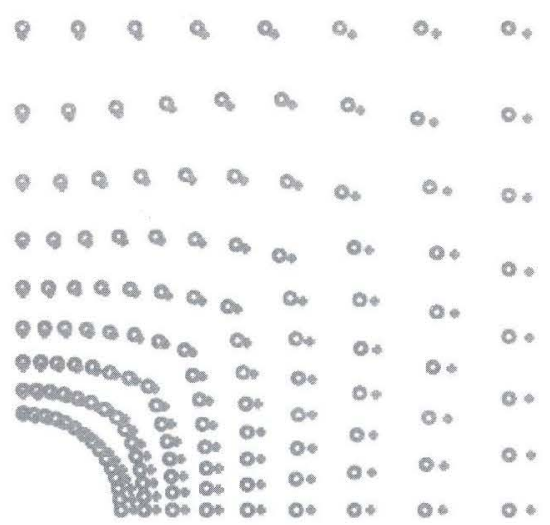

Fig. 12. Displacement of nodes with EFG method

The figure shows that, as the number of the node increases, the results obtained are closer to the analytical solution

Our numerical examination of the relationship between the density of field nodes and background mesh for 2D stress analysis problem indicates that: the ratio of the integration points to the field nodes is around 3 to 9 . Accuracy of the stress field can be improved 


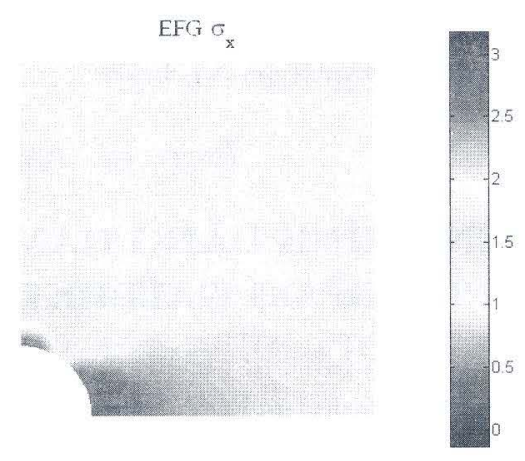

Fig. 13. EFG stress field $\sigma_{x}$

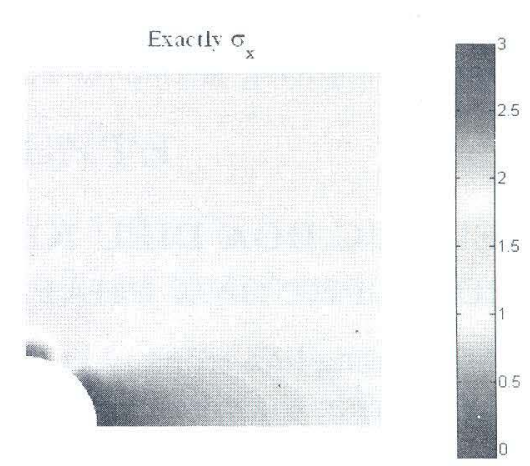

Fig. 14. Analytical stress field

efficiently by increasing the density of the field nodes, together with sufficient density of the background mesh.

The solution by the EFG method seems accurate enough and converges to the analytical solution when the number of nodes approaches infinity.

\section{CONCLUSION AND DISCUSSION}

An element free Galerkin method was implemented in Matlab for linear statics problem. The solution by this method seems accurate enough and converge to the analytical solution when the number of nodes approaches infinity.

The main advantage of the penalty method is that it leads to a positive definited and banded stiffness matrix. The stiffness matrix also has a smaller dimension than those using Lagrangian multipliers, that improves computational efficiency. Numerical examples have demonstrated the performance of the penalty method.

The EFG method is flexible with respect to the construction of the shape functions. Therefore, it is possible to improve the accuracy of the method by the choice of weight functions, by the selection of the support of EFG nodes (given by the weight function definition). The support radius of approximately 3.9 of node spacings was discovered to yield good results for all the problems studied.

\section{REFERENCES}

[1] T. Belystchko, Y. Krongauz and D. Ogan and M. Fleming and P. Krysl, Meshless methods: An overview and recent developments, International Journal for Numerical Methods in Engineering, 1996.

[2] T. Belytschko, Y. Y. Lu and G. Gu, Element free Galerkin method, International Journal for Numerical Methods in Engineering 37 (1994) 229-256.

[3] G. R. Liu, Y. T. Gu, Y. L. Wu, A Meshfree Weak-Strong-form (MWS) Method, International Workshop on MeshFree Methods, 2003.

[4] G. R. Liu, Meshfree Methods, Moving beyond the Finite Element Method, CRC Press: Boca Raton, 2003.

[5] H.-J. Chung, T. Belytschko, An Error Estimate In The EFG Method. Computational Mechanics 21 (1998) 91-100 Springer-Verlag. 
[6] J. Dolbow, T. Belytschko, Numerical Integration of The Galerkin Weak Form in Meshfree Methods, Computational Mechanics 23 (1999) 219-230 (Springer-Verlag).

Received June 16, 2009

\section{MỘT HIỆU LỰC HÓA ĐIỀU KIỆN BIÊN CHÍNH BẰNG HÀM PHẠT TRONG PHƯƠNG PHÁP PHẦN TỬ TỬ DO GALERKIN}

Một xấp xỉ không lưới trong phân tích bài toán đàn hồi hai chiều bằng phương pháp phần tử tự do Galerkin được giới thiệu. Phương pháp này dựa trên xấp xỉ bình phương tối thiểu động. Hàm chuyển vị $u(x)$ được xấp xỉ theo MLS thành $u^{h}(x)$. Xấp xỉ này được xây dựng bằng việc xử dụng các hàm trọng số dưới dạng hàm đa thức cơ sở. Việc chia nhỏ giống như phương pháp phần tử hữu hạn đóng vai trò lưới nền để thực hiện các tích phân số. Tuy nhiên các điều kiện biên chính phải được hiệu lực hóa bằng phương pháp hàm phạt. Kết quả thu được trong phân tích EFG cho bài toán hai chiều với hàm trọng số khác nhau được so sánh với các kết quả của phương pháp phần tử hữu hạn và phương pháp chính xác. 